\title{
Adomian Decomposition Method for the Solution of Boundary Layer Convective Heat Transfer with Low Pressure Gradient over a Flat
}

\author{
${ }^{1}$ M. Jiya, ${ }^{2}$ Oyubu J. \\ ${ }^{1,2}$ (Mathematics/Statistics Department, Federal University of Technology, Minna, Nigeria)
}

\begin{abstract}
This research work is aimed at obtaining solutions to the boundary layer convective heat transfer with viscous dissipation and low pressure gradient over a flat plate. The nonlinear equations of momentum and energy are transformed from partial differential equations into ordinary differential equations by using similarity transformation; the resulting equations were solved using Adomian decomposition method (ADM). An attempt was made to show the potentials and wide-range of applications of the Adomian decomposition method in the comparison with other methods used in solving heat transfer problems. The results were compared with numerical method (NM) and Homotopy perturbation method (HPM). It was observed from results obtained, that the ADM provides highly precise numerical solution for nonlinear differential equations. The results were accurate especially for $\boldsymbol{\eta} \leq \mathbf{4}$ using this method, a general equating terms of Eckert number $(E C)$ and pressure gradient $(\lambda)$ is derived which can be used to investigate velocity and temperature profiles.
\end{abstract}

Keywords - Adomian Decomposition Method (ADM), Boundary Layer, Convective Heat Transfer, Nonlinear, Pressure Gradient

\section{Introduction}

There are few phenomena in the fields of science and engineering that occurs linearly, most occur nonlinearly. Nonlinear Equations are difficult to handle, some nonlinear equations can be solved using numerical techniques [1], while some of them are solved using the analytical method such as perturbation technique, HPM and HAM [2 - 4]. [5] Study Boundary Layer Convective Heat Transfer with Low Pressure Gradient over a flat plate and they uses the method of Homotopy perturbation method. The other well-known method is Adomian's decomposition method (ADM) [6 - 7]. The ADM was used to solve a wide range of physical problems. This method provides a direct scheme for solving linear and nonlinear deterministic and stochastic equations without the need for linearization and yields convergent series solutions rapidly. Several researchers have used ADM to solve a wide range of physical problems in various engineering fields such as fluid flow and heat and mass transfer [8-9]. In this paper, The Boundary Layer Convective Heat Transfer with viscous dissipation and Low Pressure Gradient over a Flat Plate is presented and Adomian decomposition method (ADM) is employed to calculate an approximation to the solution of the system of nonlinear differential equations governing the problem, the result is achieved by implementing the ADM to determine effects of Eckert, prandtl number and pressure gradient on velocity profile and temperature and compare with other solutions.

\section{Materials and methods}

\section{Adomian Decomposition Method}

In the ADM a differential equation of the following form is considered

$L u+R u+N u=g(x)$

Where $L$ is the linear operator which is the highest order derivatives, $R$ is the remainder of linear operator including derivatives of less order than $L, N u$ represents the nonlinear terms and $g$ is the source term. Applying the inverse operator $L^{-1}$ and rearranging gives

$u=L^{-1} g(x)-L^{-1}(R u)-L^{-1}(N u)$

After integrating the source term and combining it with the terms arising from given conditions, a function $f(x)$ is defined in the equation as

$$
u=f(x)-L^{-1}(R u)-L^{-1}(N u)
$$

The nonlinear operator $N u=f(u)$ is represented by an infinite series of Adomian polynomials for the specific nonlinearity. Assuming $N u$ is analytic we write 


$$
F(u)=\sum_{n=0}^{\infty} A_{n}
$$

The polynomials $A_{n}$ 's are generated for all kinds of nonlinearity so that they depend only on $u_{0}$ to $u_{k}$ components and can be produced by the following Algorithm.

$$
\begin{aligned}
& A_{0}=f\left(u_{0}\right) \\
& A_{1}=u_{1}\left(\frac{d}{d u_{0}}\right) \mathrm{f}\left(u_{0}\right) \\
& A_{2}=u_{2}\left(\frac{d}{d u_{0}}\right) \mathrm{f}\left({ }_{0}\right) u+\frac{u_{1}^{2}}{2} \frac{d^{2}}{d u_{0}^{2}} \mathrm{f}\left(u_{0}\right)
\end{aligned}
$$

The solution $u(x)$ is defined by the following series

$$
u=\sum_{n=0}^{\infty} u_{n}
$$

Where the components are determined recursively as follows;

$u(0)=f(x)$

$u_{n+1}=-L^{-1}\left(R u_{n}\right)-L^{-1} \geq 0$

\section{Governing Equations}

3. Boundary layer convective heat transfer with low pressure Gradient over a flat plate

Consider boundary layer flow over a flat plate governed by continuity and Navier-stokes equations.

Under the boundary layer assumptions and a constant stokes equations property assumption, the continuity and Navier-stokes equations become:

Continuity equation:

$$
\frac{\partial u}{\partial x}+\frac{\partial v}{\partial y}=0
$$

Momentum equation:

$u \frac{\partial u}{\partial x}+v \frac{\partial u}{\partial y}=-\frac{1}{\rho} \frac{d p}{d x}+v \frac{\partial^{2} u}{\partial y^{2}}+g \beta\left(T-T_{\infty}\right)$

Energy equation:

$$
u \frac{\partial T}{\partial x}+v \frac{\partial T}{\partial y}=\alpha \frac{\partial^{2} T}{\partial y^{2}}+\frac{v}{c p}\left(\frac{\partial u}{\partial y}\right)^{2}
$$

Subject to the following boundary conditions:

$$
\begin{array}{lrr}
\mathrm{U}=0, \mathrm{v}=0, & \mathrm{~T}=T_{W} & \text { at } \mathrm{y}=0 \\
\mathrm{U} \rightarrow U_{\infty}, & \mathrm{T} \rightarrow T_{\infty} & \text { as } \mathrm{y} \rightarrow \infty
\end{array}
$$

By similarity transform of each of the terms in equations (3.2) to (3.3), we have;

$\eta=y \sqrt{\frac{u_{\infty}}{v x}}$

$a=\sqrt{\frac{u_{\infty}}{v}}$

$b=\sqrt{v u_{\infty}}$

$a$ and $b$ are constant of $\eta$ and $f(\eta)$,

$\eta=a \frac{y}{\sqrt{x}}, \varphi=b \sqrt{x} f(\eta), \theta(\eta)=\frac{T-T_{\infty}}{T_{W}-T_{\infty}}$

Introducing stream function, $u=\frac{\partial \varphi}{\partial y}$ and $v=-\frac{\partial \varphi}{\partial x}$

The equations can be reduced to two equations where $f$ and $\lambda$ are related to pressure gradient and $u$ the velocity by

$$
\begin{aligned}
& \lambda=-\frac{1}{\boldsymbol{\rho}} \frac{x}{u_{\infty}} \frac{d p}{d x} \quad \text { and } f^{\prime}=\frac{u}{u_{\infty}} \\
& f^{\prime \prime \prime}+\frac{1}{2} f f^{\prime \prime}=-\lambda \\
& \frac{1}{p r} \theta^{\prime \prime}+E C\left(f^{\prime \prime}\right)^{2}+\frac{f \theta^{\prime}}{2}=0 \\
& \text { Where } E C=\frac{\left(u_{0} x\right)^{2}}{c p} \frac{1}{\left(T_{W}-T_{\infty}\right)}
\end{aligned}
$$


Boundary conditions:

$f(0)=0, f^{\prime}(0)=0, \theta(0)=1, \quad f^{\prime}(\infty)=1, \theta(\infty)=0$

3.1 Adomian decomposition method solution to the equations

$f^{\prime \prime \prime}=-\frac{1}{2} f f^{\prime \prime}-\lambda$

$\theta^{\prime \prime}=-\operatorname{pr}\left(f^{\prime \prime}\right)^{2}-\frac{1}{2} p r f \theta^{\prime}$

$\frac{d 3 f}{d \eta 3}=f^{\prime \prime \prime}$, where $L_{1}=\frac{d^{3}}{d \eta^{3}}$ Then $L f=f^{\prime \prime \prime}$

$\frac{d^{2} \theta}{d \eta^{2}}=\theta^{\prime \prime} \quad$, where $L_{2}=\frac{d^{2}}{d \eta^{2}}$ Then $L \theta=\theta^{\prime \prime}$

Therefore the equations (3.17) and (3.18) become,

$L f=-\frac{1}{2} f f^{\prime \prime}-\lambda$

$L \theta=-\operatorname{ECpr}\left(f^{\prime \prime}\right)^{2}-\frac{1}{2} \operatorname{prf} \theta^{\prime}$

By (ADM)

Multiply throughout by inverse of $L$ we obtain,

$L_{1}^{-1} L f=-\frac{1}{2} L_{1}^{-1} f f^{\prime \prime}-L_{1}^{-1} \lambda$

$L_{2}^{-1} L \theta=-L_{2}^{-1} E C p r f^{\prime \prime 2}-L_{2}^{-1} \frac{1}{2} p r f \theta^{\prime}$

Where $L_{1}^{-1}=\iiint(). d \eta d \eta d \eta$ and $L_{2}^{-1}=\iint(). d \eta d \eta$

$L_{1}^{-1} L f=f-f(0)-\eta f^{\prime}(0)-\frac{\eta^{2}}{2} f^{\prime \prime}(0)$

$L_{2}^{-1} L \theta=\theta-\theta(0)-\eta \theta^{\prime}(0)$

From equation (3.24) and (3.25) we obtain

$f=f(0)+\eta f^{\prime}(0)+\frac{\eta^{2}}{2} f^{\prime \prime}(0)-\frac{1}{2} L_{1}^{-1}\left(f f^{\prime}\right)-L_{1}^{-1}(\lambda)$

$\theta=\theta(0)+\eta \theta^{\prime}(0)-E C p r L_{2}^{-1}\left(f^{\prime \prime}\right)^{2}-\frac{1}{2} p r L_{2}^{-1}\left(f \theta^{\prime}\right)$

By ADM we have,

$f(\eta)=\sum_{m=0}^{\infty} F m=f_{0}+f_{1}+f_{2}+\cdots+f_{m}$

$\theta(\eta)=\sum_{n=0}^{\infty} \theta n=\theta_{0}+\theta_{1}+\theta_{2}+\cdots+\theta_{n}$

Boundary conditions:

$f(0)=0, f^{\prime}(0)=0, \theta(0)=1, f^{\prime}(\infty)=1, \theta(\infty)=0$

$f^{\prime \prime}(0)=\alpha$ and $\theta^{\prime}(0)=\beta$ are assume value.

Applying equation (3.30) to (3.31) into (3.26) and (3.27) comparing with equation (3.28) and (3.29) we obtain,

$f_{0}(\eta)=\frac{\eta^{2}}{2} \alpha-\lambda \frac{\eta^{3}}{6}$

$\theta_{0}(\eta)=1+\eta \beta$

$f_{m+1}(\eta)=-\frac{1}{2} L_{1}^{-1} \sum_{m=0}^{\infty} A_{m}$

$\theta_{n+1}(\eta)=L_{2}^{-1} \sum_{n=0}^{\infty} B_{n}+L_{2}^{-1} \sum_{n=0}^{\infty} C_{n} \quad \mathrm{n}=0,1,2 \ldots$

Using the polynomial scheme, we obtain:

$A_{0}=f_{0} f_{0}^{\prime \prime}$

$A_{1}=f_{1} f_{0}^{\prime \prime}+f_{0} f_{1}^{\prime \prime}$

$A_{2}=f_{2} f_{0}^{\prime \prime}+f_{1} f_{1}^{\prime \prime}+f_{0} f_{2}^{\prime \prime}$

$A_{3}=f_{3} f_{0}^{\prime \prime}+f_{2} f_{1}^{\prime \prime}+f_{1} f_{2}^{\prime \prime}+f_{0} f_{3}^{\prime \prime}$

$B_{0}=\left(f_{0}^{\prime \prime}\right)^{2}$

$C_{0}=f_{0} \theta_{0}^{\prime}$

$B_{1}=2 f_{0}^{\prime \prime} f_{1}^{\prime \prime}$

$C_{1}=f_{0} \theta_{1}^{\prime}+f_{1} \theta_{0}^{\prime}$

$B_{2}=\left(f_{1}^{\prime \prime}\right)^{2}+2 f_{0}^{\prime \prime} f_{2}^{\prime \prime}$

$C_{2}=f_{0} \theta_{2}^{\prime}+f_{1} \theta_{1}^{\prime \prime}+f_{2} \theta_{0}^{\prime}$ 
By ADM we obtain:

Using maple software into equation (3.34) and (3.35) we obtain,

$f_{0}(\eta)=\frac{1}{2} \eta^{2} \alpha-\frac{1}{6} \lambda \eta^{3}$

$f_{1}(\eta)=-\frac{1}{2520} \lambda^{2} \eta^{7}+\frac{1}{360} \alpha \lambda \eta^{6}-\frac{1}{240} \alpha^{2} \eta^{5}$

$f_{2}(\eta)=-\frac{1}{623700} \lambda^{3} \eta^{11}+\frac{1}{56700} \alpha \lambda^{2} \eta^{10}-\frac{1}{16128} \alpha^{2} \lambda \eta^{9}+\frac{11}{16128} \alpha^{3} \eta^{8}$

$f_{3}(\eta)=-\frac{563}{81729648000} \lambda^{4} \eta^{15}+\frac{563}{5448643200} \alpha \lambda^{3} \eta^{14}-\frac{7081}{12454041600} \alpha^{2} \lambda^{2} \eta^{13}+\frac{577}{425779200} \alpha^{3} \lambda \eta^{12}-$ $\frac{5}{4257792} \alpha^{4} \eta^{11}$

and finally we obtain

$f(\eta)=\frac{1}{2} \eta^{2} \alpha-\frac{1}{6} \lambda \eta^{3}-\frac{1}{2520} \lambda^{2} \eta^{7}+\frac{1}{360} \alpha \lambda \eta^{6}-\frac{1}{240} \alpha^{2} \eta^{5}-\frac{1}{623700} \lambda^{3} \eta^{11}+\frac{1}{56700} \alpha \lambda^{2} \eta^{10}-$ $\frac{1}{16128} \alpha^{2} \lambda \eta^{9}+\frac{11}{16128} \alpha^{3} \eta^{8}-\frac{563}{81729648000} \lambda^{4} \eta^{15}+\frac{563}{5448643200} \alpha \lambda^{3} \eta^{14}-\frac{7081}{12454041600} \alpha^{2} \lambda^{2} \eta^{13}+$ $\frac{577}{425779200} \alpha^{3} \lambda \eta^{12}-\frac{5}{4257792} \alpha^{4} \eta^{11}$

$\theta_{0}(\eta)=1+\eta \beta$

$\theta_{1}(\eta)=-\frac{1}{2} \mathrm{E} C p r \alpha^{2} \eta^{2}+\frac{1}{3} \mathrm{E} C p r \eta^{3} \lambda-\frac{1}{12} \mathrm{E} C p r \eta^{4} \lambda^{2}-\frac{1}{48} \beta p r \alpha \eta^{4}+\frac{1}{240} \beta p r \lambda \eta^{5}$

$\theta_{2}(\eta)=-\frac{1}{1680} \mathrm{E} C p r \lambda^{3} \eta^{3}+\frac{1}{210} \mathrm{E} C p r \alpha \lambda^{2} \eta^{7}-\frac{1}{90} \alpha^{2} \mathrm{E} C p r \lambda \eta^{6}+\frac{1}{120} \alpha^{3} \mathrm{E} C p r \eta^{5}+$ $\frac{1}{41472} \lambda^{2} \eta^{9} \beta p r^{2}-\frac{1}{4608} \eta^{8} \alpha \beta \lambda p r^{2}-\frac{1}{2016} \mathrm{E} C p r^{2} \lambda^{3} \eta^{8}+\frac{1}{252} \mathrm{E} C p r^{2} \alpha \lambda^{2} \eta^{7}+\frac{1}{2016} \eta^{7} \beta \alpha^{2} p r^{2}-$ $\frac{1}{90} \alpha^{2} \mathrm{E} C p r^{2} \lambda \eta^{6}+\frac{1}{80} \alpha^{3} \mathrm{E} C p r^{2} \eta^{5}+\frac{1}{362880} \lambda^{2} \beta p r \eta^{9}-\frac{1}{40320} \eta^{8} \alpha \beta \lambda p r+\frac{1}{20160} \eta^{7} \beta \alpha^{2} p r$

$\theta_{3}(\eta)=\frac{1}{194594400} \lambda^{3} \beta p r \eta^{13}-\frac{1}{672} \alpha^{4} \mathrm{E} C p r \eta^{8}-\frac{13}{2721600} \operatorname{ECpr} \lambda^{4} \eta^{12}+\frac{1}{25159680} \lambda^{3} p r^{2} \eta^{13} \beta-$ $\frac{1}{285120} \eta^{12} \mathrm{E} C p r^{2} \lambda^{4}-\frac{11}{2903040} \eta^{10} \beta \alpha^{3} p r-\frac{1}{345600} \eta^{10} \beta \alpha^{3} p r^{2}-\frac{1}{4480} \alpha^{4} \eta^{8} \mathrm{E} C p r^{2}+$ $\frac{25}{18144} \alpha^{3} \mathrm{E} C p r \lambda \eta^{9}+\frac{13}{226800} \mathrm{E} C p r \lambda^{3} \eta^{11} \alpha-\frac{11}{45360} \mathrm{E} C p r \lambda^{2} \eta^{10} \alpha^{2}-\frac{1}{1935360} \eta^{12} \alpha p r^{2} \lambda^{2} \beta-$ $\frac{1}{14968800} \eta^{12} \alpha \lambda^{2} \beta p r+\frac{23}{10644480} \eta^{11} \alpha^{2} \beta \lambda p r^{2}+\frac{1}{23760} \eta^{11} \alpha \mathrm{E} C p r^{2} \lambda^{3}+\frac{1}{3548160} \eta^{11} \alpha^{2} \beta \lambda p r-$ $\frac{163}{907200} \eta^{10} \lambda^{2} \mathrm{E} C p r^{2} \alpha^{2}+\frac{17}{51840} \eta^{9} \alpha^{3} \mathrm{E} C p r^{2} \lambda-\frac{1}{663552} \eta^{12} \alpha p r^{3} \lambda^{2} \beta+\frac{1}{152064} \eta^{11} \alpha^{2} \beta \lambda p r^{3}+$ $\frac{1}{33264} \eta^{11} \alpha \mathrm{E} C p r^{3} \lambda^{3}-\frac{1}{7200} \eta^{10} \lambda^{2} \mathrm{E} C p r^{3} \alpha^{2}+\frac{1}{23040} \eta^{9} \alpha^{3} \mathrm{E} C p r^{3} \lambda+\frac{1}{8626176} p r^{3} \lambda^{3} \eta^{13} \beta-$ $\frac{1}{399168} \eta^{12} \mathrm{E} C p r^{3} \lambda^{4}-\frac{1}{103680} \eta^{10} p r^{3} \beta \alpha^{3}-\frac{1}{3584} \alpha^{4} \eta^{8} \mathrm{E} C p r^{3}$

and finally we obtain

$\theta(\eta)=1-\frac{1}{2} \mathrm{E} C \operatorname{pr} \alpha^{2} \eta^{2}-\frac{1}{12} \mathrm{E} C p r \eta^{4} \lambda^{2}-\frac{1}{48} \beta p r \alpha \eta^{4}+\frac{1}{240} \beta p r \lambda \eta^{5}+\eta \beta+\frac{1}{3} \mathrm{E} C p r \eta^{3} \lambda-$ $\frac{1}{90} \alpha^{2} \mathrm{E} C p r \lambda \eta^{6}+\frac{1}{210} \mathrm{E} C p r \alpha \lambda^{2} \eta-\frac{1}{40320} \eta^{8} \alpha \beta \lambda p r^{7}-\frac{1}{4608} \eta^{8} \alpha \beta \lambda p r^{2}+\frac{1}{252} \mathrm{E} C p r^{2} \alpha \lambda^{2} \eta^{7}-$ $\frac{1}{90} \alpha^{2} \mathrm{E} C p r^{2} \lambda \eta^{6}+\frac{1}{362880} \lambda^{2} \beta p r \eta^{9}+\frac{1}{120} \alpha^{3} \mathrm{E} C p r \eta^{5}-\frac{1}{1680} \mathrm{E} C p r \lambda^{3} \eta^{3}+\frac{1}{20160} \eta^{7} \beta \alpha^{2} p r+$ $\frac{1}{41472} \lambda^{2} \eta^{9} \beta p r^{2}-\frac{1}{2016} \mathrm{E} C p r^{2} \lambda^{3} \eta^{8}+\frac{1}{2016} \eta^{7} \beta \alpha^{2} p r^{2}+\frac{1}{80} \alpha^{3} \mathrm{E} C p r^{2} \eta^{5}+$ $\frac{1}{194594400} \lambda^{3} \beta p r \eta^{13}-\frac{1}{672} \alpha^{4} \mathrm{E} C p r \eta^{8}-\frac{13}{2721600} \mathrm{E} C p r \lambda^{4} \eta^{12}+\frac{1}{25159680} \lambda^{3} p r^{2} \eta^{13} \beta-$ $\frac{1}{285120} \eta^{12} \mathrm{E} C p r^{2} \lambda^{4}-\frac{11}{2903040} \eta^{10} \beta \alpha^{3} p r-\frac{1}{345600} \eta^{10} \beta \alpha^{3} p r^{2}-\frac{1}{4480} \alpha^{4} \eta^{8} \mathrm{E} C p r^{2}+$ $\frac{25}{18144} \alpha^{3} \mathrm{E} C p r \lambda \eta^{9}+\frac{13}{226800} \mathrm{E} C p r \lambda^{3} \eta^{11} \alpha-\frac{11}{45360} \mathrm{E} C p r \lambda^{2} \eta^{10} \alpha^{2}-\frac{1}{1935360} \eta^{12} \alpha p r^{2} \lambda^{2} \beta-$ $\frac{1}{14968800} \eta^{12} \alpha \lambda^{2} \beta p r+\frac{23}{10644480} \eta^{11} \alpha^{2} \beta \lambda p r^{2}+\frac{1}{23760} \eta^{11} \alpha \mathrm{E} C p r^{2} \lambda^{3}+\frac{1}{3548160} \eta^{11} \alpha^{2} \beta \lambda p r-$ $\frac{163}{907200} \eta^{10} \lambda^{2} \mathrm{E} C p r^{2} \alpha^{2}+\frac{17}{51840} \eta^{9} \alpha^{3} \mathrm{E} C p r^{2} \lambda-\frac{1}{663552} \eta^{12} \alpha p r^{3} \lambda^{2} \beta+\frac{1}{152064} \eta^{11} \alpha^{2} \beta \lambda p r^{3}+$ 
$\frac{1}{33264} \eta^{11} \alpha \mathrm{E} C p r^{3} \lambda^{3}-\frac{1}{7200} \eta^{10} \lambda^{2} \mathrm{E} C p r^{3} \alpha^{2}+\frac{1}{23040} \eta^{9} \alpha^{3} \mathrm{E} C p r^{3} \lambda+\frac{1}{8626176} p r^{3} \lambda^{3} \eta^{13} \beta-$ $\frac{1}{399168} \eta^{12} \mathrm{E} C p r^{3} \lambda^{4}-\frac{1}{103680} \eta^{10} p r^{3} \beta \alpha^{3}-\frac{1}{3584} \alpha^{4} \eta^{8} \mathrm{E} C p r^{3}$

\section{Results And Discussions}

Adomian decomposition method solution to boundary layer convective heat transfer low pressure gradient over a flat plate are obtained for the various values of physical parameters involve in the problem such as prandtl number, Ecket number and pressure gradient. When there is no pressure gradient, variation of the measuring parameters are compared with that of HPM [10] and NM [11] methods. The two assumed values taken are $\alpha=0.332057$ and $\beta=-0.3487661$

Table 4.1 the results of ADM, NM and $\operatorname{HPM}$ for $\mathbf{F}^{\prime}(\eta)$ at $\lambda=0$

\begin{tabular}{lrrr}
\hline $\boldsymbol{\eta}$ & ADM & NM & HPM \\
\hline $\mathbf{0 . 0}$ & 0 & 0 & 0 \\
$\mathbf{0 . 2}$ & 0.066408 & 0.066408 & 0.069907 \\
$\mathbf{0 . 4}$ & 0.132764 & 0.132764 & 0.139764 \\
$\mathbf{0 . 6}$ & 0.198937 & 0.198937 & 0.209441 \\
$\mathbf{0 . 8}$ & 0.264709 & 0.264709 & 0.278723 \\
$\mathbf{1 . 0}$ & 0.32978 & 0.32978 & 0.347312 \\
$\mathbf{1 . 2}$ & 0.393776 & 0.393776 & 0.414831 \\
$\mathbf{1 . 4}$ & 0.456261 & 0.456262 & 0.480832 \\
$\mathbf{1 . 6}$ & 0.516756 & 0.516757 & 0.544806 \\
$\mathbf{1 . 8}$ & 0.574755 & 0.574758 & 0.606195 \\
$\mathbf{2 . 0}$ & 0.629756 & 0.629766 & 0.664414 \\
$\mathbf{2 . 2}$ & 0.68128 & 0.68131 & 0.718871 \\
$\mathbf{2 . 4}$ & 0.728891 & 0.728982 & 0.768993 \\
$\mathbf{2 . 6}$ & 0.772204 & 0.772455 & 0.814261 \\
$\mathbf{2 . 8}$ & 0.810869 & 0.81151 & 0.854239 \\
$\mathbf{3 . 0}$ & 0.844521 & 0.846044 & 0.888611 \\
$\mathbf{3 . 2}$ & 0.872672 & 0.876044 & 0.917222 \\
$\mathbf{3 . 4}$ & 0.894529 & 0.876081 & 0.940107 \\
$\mathbf{3 . 6}$ & 0.90871 & 0.92333 & 0.957524 \\
$\mathbf{3 . 8}$ & 0.912811 & 0.941118 & 0.969974 \\
\hline & & &
\end{tabular}


Table 4.2. Results of ADM method for $F^{\prime}(\eta)$ for different pressure gradient

\begin{tabular}{rrrr}
\hline $\boldsymbol{\eta}$ & $\lambda=\mathbf{0 . 0 0 0 0}$ & $\lambda=-0.0018$ & \multicolumn{1}{c}{$\boldsymbol{\lambda}=0.010$} \\
\hline $\mathbf{0}$ & 0 & 0 & 0 \\
$\mathbf{0 . 2}$ & 0.066408 & 0.066444 & 0.066208 \\
$\mathbf{0 . 4}$ & 0.132764 & 0.132908 & 0.131965 \\
$\mathbf{0 . 6}$ & 0.198938 & 0.199261 & 0.197142 \\
$\mathbf{0 . 8}$ & 0.264717 & 0.26529 & 0.261535 \\
$\mathbf{1}$ & 0.32982 & 0.33071 & 0.324874 \\
$\mathbf{1 . 2}$ & 0.39392 & 0.395191 & 0.386854 \\
$\mathbf{1 . 4}$ & 0.456685 & 0.458397 & 0.447172 \\
$\mathbf{1 . 6}$ & 0.517834 & 0.520038 & 0.505585 \\
$\mathbf{1 . 8}$ & 0.577216 & 0.579955 & 0.561988 \\
$\mathbf{2}$ & 0.6349 & 0.638207 & 0.616507 \\
$\mathbf{2 . 2}$ & 0.691304 & 0.6952 & 0.66962 \\
$\mathbf{2 . 4}$ & 0.747322 & 0.751816 & 0.722284 \\
$\mathbf{2 . 6}$ & 0.80448 & 0.809569 & 0.776092 \\
$\mathbf{2 . 8}$ & 0.865091 & 0.870757 & 0.833426 \\
$\mathbf{3}$ & 0.932407 & 0.938614 & 0.897616 \\
\hline
\end{tabular}

Table 4.3: The ADM method for $\theta(\eta)$ for prandtl no. $(\mathrm{pr}=1.0)$ and Pressure gradient $\lambda=0$ and for $E C=0.0$

\begin{tabular}{lrll}
\hline $\boldsymbol{\eta}$ & \multicolumn{1}{l}{ ADM } & NM & HPM \\
\hline $\mathbf{0 . 0}$ & 0.930251 & $\mathbf{1}$ & $\mathbf{1}$ \\
$\mathbf{0 . 2}$ & 0.860555 & $\mathbf{0 . 9 3 3 5 9}$ & $\mathbf{0 . 9 3 0 0 9}$ \\
$\mathbf{0 . 4}$ & 0.791052 & $\mathbf{0 . 8 6 7 2 4}$ & $\mathbf{0 . 8 6 0 2 4}$ \\
$\mathbf{0 . 6}$ & 0.721971 & $\mathbf{0 . 8 0 1 0 6}$ & $\mathbf{0 . 7 9 0 5 6}$ \\
$\mathbf{0 . 8}$ & 0.653626 & $\mathbf{0 . 7 3 5 2 9}$ & $\mathbf{0 . 7 2 1 2 8}$ \\
$\mathbf{1 . 0}$ & 0.58641 & $\mathbf{0 . 6 7 0 2 2}$ & $\mathbf{0 . 6 5 2 6 9}$ \\
$\mathbf{1 . 2}$ & 0.520781 & $\mathbf{0 . 6 0 6 2 2}$ & $\mathbf{0 . 5 8 5 1 7}$ \\
$\mathbf{1 . 4}$ & 0.457246 & $\mathbf{0 . 5 4 3 7 4}$ & $\mathbf{0 . 5 1 9 1 7}$ \\
$\mathbf{1 . 6}$ & 0.396339 & $\mathbf{0 . 4 8 3 2 4}$ & $\mathbf{0 . 4 5 5 1 9}$ \\
$\mathbf{1 . 8}$ & 0.338599 & $\mathbf{0 . 4 2 5 2 4}$ & $\mathbf{0 . 3 9 3 8 1}$ \\
$\mathbf{2 . 0}$ & 0.284554 & $\mathbf{0 . 3 7 0 2 3}$ & $\mathbf{0 . 3 3 5 5 9}$ \\
$\mathbf{2 . 2}$ & 0.234708 & $\mathbf{0 . 3 1 8 6 9}$ & $\mathbf{0 . 2 8 1 1 3}$ \\
$\mathbf{2 . 4}$ & 0.189554 & $\mathbf{0 . 2 7 1 0 2}$ & $\mathbf{0 . 2 3 1 0 1}$ \\
$\mathbf{2 . 6}$ & 0.149618 & $\mathbf{0 . 2 2 7 5 5}$ & $\mathbf{0 . 1 8 5 7 4}$ \\
$\mathbf{2 . 8}$ & 0.115554 & $\mathbf{0 . 1 8 8 4 9}$ & $\mathbf{0 . 1 4 5 7 6}$ \\
$\mathbf{3 . 0}$ & 0.088318 & $\mathbf{0 . 1 4 3 9 6}$ & $\mathbf{0 . 1 1 1 3 9}$ \\
$\mathbf{3 . 2}$ & 0.069448 & $\mathbf{0 . 1 2 3 9 2}$ & $\mathbf{0 . 0 8 2 7 8}$ \\
$\mathbf{3 . 4}$ & 0.061485 & $\mathbf{0 . 0 8 8 2 4}$ & $\mathbf{0 . 0 5 9 8 9}$ \\
$\mathbf{3 . 6}$ & & $\mathbf{0 . 0 6 6 6 7}$ & $\mathbf{0 . 0 4 2 4 8}$ \\
\hline & & &
\end{tabular}


Table 4.4. Result of ADM method for $\theta(\eta)$ for prandtl number, pressure gradient at $\lambda=0$ and $E C=0.0$

\begin{tabular}{rrrrr}
\hline $\boldsymbol{\eta}$ & \multicolumn{1}{c}{ pr=0.5 } & \multicolumn{1}{c}{ pr=1.0 } & \multicolumn{1}{c}{ pr=1.5 } \\
\hline $\mathbf{0 . 0}$ & 1 & 1 & 1 \\
$\mathbf{0 . 2}$ & 0.930249 & 0.930251 & 0.930253 \\
$\mathbf{0 . 4}$ & 0.860524 & 0.860555 & 0.860586 \\
$\mathbf{0 . 6}$ & 0.790897 & 0.791052 & 0.791208 \\
$\mathbf{0 . 8}$ & 0.72148 & 0.721971 & 0.72246 \\
$\mathbf{1 . 0}$ & 0.652435 & 0.653626 & 0.654808 \\
$\mathbf{1 . 2}$ & 0.583962 & 0.58641 & 0.588825 \\
$\mathbf{1 . 4}$ & 0.516303 & 0.520781 & 0.525164 \\
$\mathbf{1 . 6}$ & 0.449732 & 0.457246 & 0.464526 \\
$\mathbf{1 . 8}$ & 0.384552 & 0.396339 & 0.407614 \\
$\mathbf{2 . 0}$ & 0.321087 & 0.338599 & 0.355098 \\
$\mathbf{2 . 2}$ & 0.259676 & 0.284554 & 0.307592 \\
$\mathbf{2 . 4}$ & 0.20067 & 0.234708 & 0.265659 \\
$\mathbf{2 . 6}$ & 0.144429 & 0.189554 & 0.229887 \\
$\mathbf{2 . 8}$ & 0.091329 & 0.149618 & 0.201056 \\
$\mathbf{3 . 0}$ & 0.041784 & 0.115554 & 0.180464 \\
\hline
\end{tabular}

Table 4.5. The results of ADM method of $\theta(\eta)$ and different prandtl numbers for pressure gradient $(\lambda=0.1111)$ and $\mathrm{EC}=0.0$

\begin{tabular}{lrrr}
\hline$\eta$ & \multicolumn{1}{c}{ pr=0.5 } & pr=1.0 & \multicolumn{1}{l}{ pr=1.5 } \\
\hline $\mathbf{0 . 0}$ & 1 & 1 & 1 \\
$\mathbf{0 . 2}$ & 0.930249 & 0.930251 & 0.930252 \\
$\mathbf{0 . 4}$ & 0.860524 & 0.860554 & 0.860584 \\
$\mathbf{0 . 6}$ & 0.79089 & 0.79104 & 0.791189 \\
$\mathbf{0 . 8}$ & 0.721454 & 0.721919 & 0.722382 \\
$\mathbf{1 . 0}$ & 0.652355 & 0.653467 & 0.654572 \\
$\mathbf{1 . 2}$ & 0.583765 & 0.586021 & 0.588249 \\
$\mathbf{1 . 4}$ & 0.51588 & 0.519955 & 0.523952 \\
$\mathbf{1 . 6}$ & 0.448919 & 0.455673 & 0.462242 \\
$\mathbf{1 . 8}$ & 0.383111 & 0.393586 & 0.403668 \\
$\mathbf{2 . 0}$ & 0.318696 & 0.334103 & 0.348746 \\
$\mathbf{2 . 2}$ & 0.255917 & 0.277612 & 0.297942 \\
$\mathbf{2 . 4}$ & 0.195022 & 0.224487 & 0.251678 \\
$\mathbf{2 . 6}$ & 0.136264 & 0.175092 & 0.210367 \\
$\mathbf{2 . 8}$ & 0.079914 & 0.129811 & 0.174481 \\
$\mathbf{3 . 0}$ & 0.026276 & 0.089098 & 0.122006 \\
\hline
\end{tabular}


Adomian Decomposition Method for the Solution of Boundary Layer Convective Heat Transfer with

Table 4.6 the results of ADM method for $\theta(\eta)$ and different prandtl numbers for pressure gradient for $\lambda=0$ and $\mathrm{EC}=0.2$

\begin{tabular}{lrrr}
\hline$\eta$ & pr=0.5 & pr=1.0 & \multicolumn{1}{c}{ pr=1.5 } \\
\hline $\mathbf{0 . 0}$ & 1 & 1 & 1 \\
$\mathbf{0 . 2}$ & 0.930038 & 0.929829 & 0.92962 \\
$\mathbf{0 . 4}$ & 0.859718 & 0.858943 & 0.858168 \\
$\mathbf{0 . 6}$ & 0.789161 & 0.787585 & 0.786011 \\
$\mathbf{0 . 8}$ & 0.718525 & 0.716073 & 0.713631 \\
$\mathbf{1 . 0}$ & 0.648003 & 0.644797 & 0.641615 \\
$\mathbf{1 . 2}$ & 0.577813 & 0.574194 & 0.570623 \\
$\mathbf{1 . 4}$ & 0.508197 & 0.504742 & 0.501359 \\
$\mathbf{1 . 6}$ & 0.439408 & 0.436926 & 0.434524 \\
$\mathbf{1 . 8}$ & 0.371703 & 0.371225 & 0.370779 \\
$\mathbf{2 . 0}$ & 0.305335 & 0.308083 & 0.310708 \\
$\mathbf{2 . 2}$ & 0.240551 & 0.247901 & 0.254789 \\
$\mathbf{2 . 4}$ & 0.177587 & 0.191037 & 0.203411 \\
$\mathbf{2 . 6}$ & 0.116687 & 0.137827 & 0.156915 \\
$\mathbf{2 . 8}$ & 0.058121 & 0.088643 & 0.115714 \\
$\mathbf{3 . 0}$ & 0.002229 & 0.043995 & 0.080484 \\
\hline
\end{tabular}

Table 4.7 The results of ADM method for $\theta(\eta)$ and different prandtl numbers for pressure gradient for

\begin{tabular}{lrrr}
\multicolumn{4}{c}{$\lambda=\mathbf{0 . 0 2 7}$ and $\mathbf{E C}=\mathbf{0 . 0}$} \\
\hline $\boldsymbol{\eta}$ & \multicolumn{1}{c}{ pr=0.5 } & \multicolumn{1}{c}{ pr=1.0 } & pr=1.5 \\
\hline $\mathbf{0 . 0}$ & 1 & 1 & 1 \\
$\mathbf{0 . 2}$ & 0.930249 & 0.930251 & 0.930253 \\
$\mathbf{0 . 4}$ & 0.860525 & 0.860556 & 0.860587 \\
$\mathbf{0 . 6}$ & 0.790898 & 0.791055 & 0.791213 \\
$\mathbf{0 . 8}$ & 0.721486 & 0.721984 & 0.722479 \\
$\mathbf{1 . 0}$ & 0.652454 & 0.653664 & 0.654865 \\
$\mathbf{1 . 2}$ & 0.58401 & 0.586504 & 0.588964 \\
$\mathbf{1 . 4}$ & 0.516405 & 0.520981 & 0.525458 \\
$\mathbf{1 . 6}$ & 0.449929 & 0.457626 & 0.465077 \\
$\mathbf{1 . 8}$ & 0.3849 & 0.397001 & 0.408561 \\
$\mathbf{2 . 0}$ & 0.321664 & 0.339677 & 0.356614 \\
$\mathbf{2 . 2}$ & 0.26058 & 0.28621 & 0.309879 \\
$\mathbf{2 . 4}$ & 0.202023 & 0.237131 & 0.268952 \\
$\mathbf{2 . 6}$ & 0.146374 & 0.19296 & 0.234467 \\
$\mathbf{2 . 8}$ & 0.094035 & 0.15426 & 0.207313 \\
$\mathbf{3 . 0}$ & 0.04544 & 0.121747 & 0.189037 \\
$\mathbf{3 . 2}$ & 0.001101 & 0.096511 & 0.182537 \\
\hline
\end{tabular}

In order to verify the accuracy of the current study we illustrate the comparison of ADM with HPM and NM as shown in Tables 4.1, 4.2 and Table 4.3. The results are obtained for $f^{\prime}(\eta)$ and $\theta(\eta)$ with $E C=0.0$ and $\lambda=0$. The results proved that the answers for velocity and temperature distributions are of good agreement with other methods. 
Effects of prandtl number and Eckert number parameter on the temperature profile is shown in Table 4.4. Temperature increment occurs with slight increase in prandtl number, while the temperature decreases with a slight increase in Eckert number. Also when there is no pressure gradient, the results obtained employing $\mathrm{ADM}$ and compare with the result of HPM and NM for $p r=1$, and $E C=0$, the values obtained from the ADM method are of good agreement with numerical method as shown in table 4.3. While table 4.4 shows the variation of $f^{\prime}(\eta)$ with different values of pressure gradient $(\lambda)$. Figure 4.4 to 4.7 shows the effect of prandtl, Eckert number and pressure gradient, it is observed that for a fixed value of $\eta$, an increase in $\operatorname{Pr}$ number lead to a corresponding increase on the temperature, this is most pronounced on the higher values of the pressure gradient $(\lambda)$ shown in table 4.5

From Table 4.6 and 4.7 an increase in the Eckert number leads to decrease in the temperature. The effect of pressure gradient $(\lambda)$ variation on the thermal boundary layer thickness is higher than the Pr number, and the thermal boundary thickness decrease with increase in pressure gradient which its increase leads to increase in the surface temperature

\section{Conclusion}

In this paper, Adomian Decomposition Method has been successful applied to natural convection heat transfer problem with specified boundary conditions for momentum and energy equations. The results were compared with ones from Homotopy Perturbation Method and Numerical Method and the result shows excellent agreement with this methods. Also, the velocity and temperature profiles were obtained as a function of $\eta, p r$ number, and pressure gradient $\lambda$ using ADM method. An attempt has been made to show the effect of pressure gradient, $p r$ number and $E C$ number on temperature and heat transfer boundary layer. This method accelerated the convergence to solutions. The ADM provide efficient alternative tools in solving nonlinear models.

\section{Acknowledgements}

The authors are grateful to members of Staff of Mathematics/Statistics Department, Federal University of Technology, Minna for their supports during this research work.

\section{References}

[1] Hasanpour A. M., Farhadi K, Sedighi H. R. A. Shorynejad, Lattice Boltzmann Simulation for Magnetohydrodynamic Mixed Convective Flow in a Porous Medium. World Applied Science Journal 11, 2010, 1124-1132.

[2] Ganji D.D., and Rajabi A. , Application of perturbation method in nonlinear heat conduction and convection equations, Physics Letters A, 360( 4-5), 2007, 570-573

[3] Ganji D. D. , Babazadeh H., Noori F., Pirouz M. M, Janipour M., An Application of Homotopy Perturbation Method for Non-linear Blasius Equation to Boundary Layer Flow Over a Flat Plate, International Journal of Nonlinear Science, 7(4), 2009 399-404

[4] Esmaeilpour M and Ganji D. D., Application of He's homotopy perturbation method to boundary layer flow and convection heat transfer over a flat plate. Physics Letters, A372, 2007 33-38

[5] Fathizadeh .M and Aroujalian A., Study of Boundary Layer Convective Heat Transfer With Low Pressure Gradient, International Journal Chemical Engineering. 9(1), 2012,33-39

[6] Adomian, G., A review of the decomposition method in applied mathematics, Journal Mathematics. Analysis Application. 135, 1998, 501-544.

[7] Adomian G, Solving Frontier Problems of Physics: The Decomposition Method (Boston, MA Kluwer, 1994).

[8] Mirgolbabaei H., Barari A. and Ibsen L. B., Analytical Solution of Forced-Convective Boundary-Layer Flow Over a Flat Plate, Archives of Civil and Mechanical Engineering, X(2), 2010, 41-51

[9] Makinde O. D, Olajuwon B.I. , and Gbolagade A.W., Adomian Decomposition Approach to a Boundary Layer Flow with Thermal Radiation Past a Moving Vertical Porous Plate, Int. J. of Appl. Math and Mech. 3(3), 2007, 62-70

[10] Ramiar, A., Ganji, D. D. and Esmaili, Q., Homotopy perturbartion method and variational iteration method for orthogonal 2-D and axisymmetric impinging jet problems, International Journal. Nonlinear Science Numerical. Simulation, 9, 2008, 115-130

[11] Bird, B. R., Stewart, W. E. and Lightfood, E. N., Transfer phenomena. (Second ed. John Wiley and Sons, 2002). 